\title{
Post-implant analysis in permanent breast seed implant: automated plan reconstruction using simulated annealing
}

\author{
Elizabeth Watt, PhD',2, Matthew Skarsgard, BASc², Michael Roumeliotis, PhD',2,3, Siraj Husain, MD 3.4 , Tyler Meyer, PhD',2,3 \\ 'Department of Physics and Astronomy, University of Calgary, Calgary AB, Canada, ${ }^{2}$ Department of Medical Physics, Tom Baker Cancer Centre, \\ Calgary AB, Canada, ${ }^{3}$ Department of Oncology, University of Calgary, Calgary AB, Canada, ${ }^{4}$ Department of Radiation Oncology. Tom Baker \\ Cancer Centre, Calgary AB, Canada
}

\begin{abstract}
Purpose: Post-implant analysis in permanent breast seed implant (PBSI) brachytherapy is an important component of the quality assurance process that indicates dosimetric quality relevant to patient outcome, indicating salvage therapy if inadequate, as well as providing feedback to the brachytherapy team to improve future treatments. To measure geometric indices on implant quality, plan reconstruction must be performed to correlate each planned and post-implant seed location. In this work, a simulated-annealing-based algorithm is developed to perform this plan reconstruction automatically.

Material and methods: The plan reconstruction algorithm was developed in MATLAB, taking the patient pre-treatment and post-implant (Day 0) plan and associated contours as inputs. For 19 treated patients, a reconstruction was obtained that defined the correspondence between each planned and post-implant seed. The simulated-annealing algorithm was used to reconstruct each patient 10 times to assess the variability in convergence. Manual reconstructions performed by at least two independent observers to obtain consensus were defined as the ground truth; these were compared to the automatic reconstructions obtained by the algorithm. Metrics on seed placement accuracy and needle strand angulation were calculated for the patients.

Results: The algorithm performed reconstructions on 19 patients (1235 seeds) with ground-truth reconstructions, obtaining $97 \pm 8 \%$ correct matches. This strong performance indicates the ability to incorporate this algorithm into the clinical quality assurance workflow.

Conclusions: The plan reconstruction algorithm developed herein performed very well in a 19-patient cohort. This algorithm can be incorporated into the clinical process to assist in the assessment of center-specific seed placement accuracy and can be used to gather implant metrics in an automated, standardized fashion for future PBSI trials.

J Contemp Brachytherapy 2019; 11, 1: 61-68

DOI: https://doi.org/10.5114/jcb.2019.83338
\end{abstract}

Key words: permanent breast seed implant, post-implant analysis, plan reconstruction, simulated annealing.

\section{Purpose}

Accelerated partial breast irradiation (APBI) has been realized in a variety of external beam and brachytherapy modalities, with the ultimate goal of irradiating a smaller portion of the breast and requiring fewer cancer center visits for the patient than standard-of-care whole breast irradiation $[1,2,3,4,5,6]$. Permanent breast seed implant (PBSI) is a brachytherapy-based form of APBI that involves surgical insertion of ${ }^{103} \mathrm{Pd}$ seeds into the post-lumpectomy breast as a one-day outpatient procedure $[7,8]$. Clinical outcomes published at a median follow-up of 63 months found a local recurrence rate of $1.2 \pm 1.2 \%$, comparable to nomogram-based estimates for whole breast irradiation [8]. As the PBSI technique matures, numerous studies in recent literature have investigated how to optimize aspects of the treatment process, including treatment planning [9,10,11,12], training $[13,14]$, clinical practice $[15,16]$, and post-implant analysis $[17,18,19,20,21]$.

Post-implant analysis is a key component in the clinical quality assurance process for permanent implant brachytherapy procedures. This analysis traditionally focuses on the dosimetry, as this can be correlated to clinical outcome and provides insight into the decision to offer salvage radiotherapy where appropriate. Post-implant analysis can, however, be extended to provide metrics on the geometric quality of the implant relative to the
Address for correspondence: Elizabeth Watt, PhD, Tom Baker Cancer Centre, Department of Medical Physics, 1331 - 29 St. NW, Calgary AB, T2N 4N2, Canada, phone: +1 403521 3792, fax: +1 403521 3327, ๑ e-mail: elizabeth.watt@ahs.ca
Received: 04.12 .2018

Accepted: 30.01 .2019

Published: 28.02.2019 
treatment plan, including seed placement accuracy and angulation of needles. Such metrics can provide invaluable feedback to the brachytherapy team to improve the treatment technique. In current clinical practice, however, the collection of such metrics requires manual reconstruction of the implant to match each planned seed to its corresponding post-implant seed. This is a potentially user-dependent and time-consuming process; thus, it is rarely performed in routine practice.

In 2016, Morton et al. [20] reported seed placement accuracy for 20 patients treated with PBSI. Their results provided guidance to the brachytherapy community on seed placement accuracy and implant metrics; the first report on these important parameters in the context of PBSI. The first step in their work, and any investigation of implant metrics, involved plan reconstruction to match corresponding planned and post-implant seeds. This reconstruction was accomplished manually. Manual plan reconstruction can be extremely time-consuming and often requires additional software infrastructure to perform effectively, allowing the co-registered viewing of planned seed locations and identified post-implant seed locations. Automated plan reconstruction has been developed for use in permanent prostate seed implant $[22,23]$. Seed placement error in PBSI has been reported to be substantially larger $[20,22,23,24,25,26,27]$, however, rendering the development of reconstruction algorithms specifically for this site an important clinical investigation. The development of an automated plan reconstruction algorithm specific to PBSI will permit the analysis of seed placement accuracy for each patient
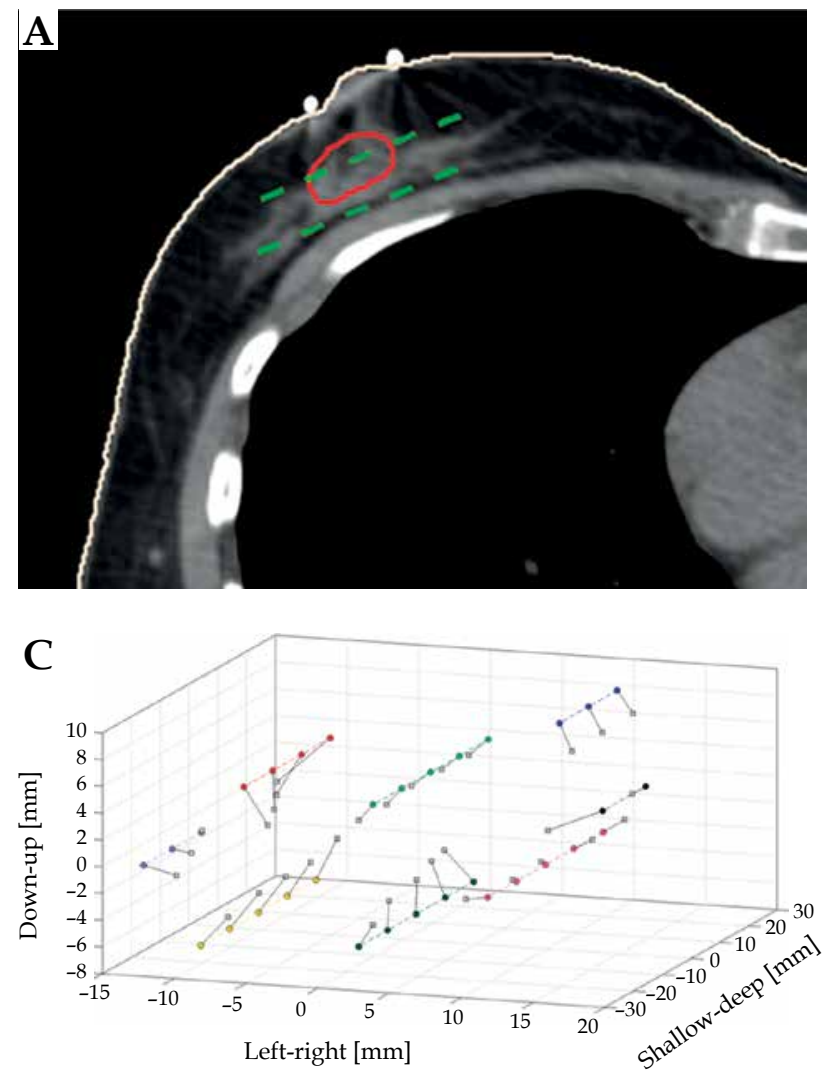

in a robust, simple manner, allowing brachytherapy teams to consistently review and improve their treatment technique. Without this algorithm, reconstructing the plan manually is the only option; a process that is extremely challenging to perform without dedicated software architecture and observer expertise and patience.

The goal in this work is to develop an automated algorithm to perform plan reconstruction for PBSI. This algorithm is developed using simulated annealing optimization. In this study, we report the accuracy of the algorithm and implant metrics for a cohort of previously treated PBSI patients from our institution.

\section{Material and methods}

\section{Permanent breast seed implant}

Patients treated with PBSI underwent a planning CT scan approximately eight to ten weeks following lumpectomy and four weeks prior to implant. This scan was taken with the patient in the treatment position, lying supine with the ipsilateral arm raised. The clinical target volume (CTV) was contoured as the seroma and surrounding fibrosis. A planning target volume (PTV) was created as a 10-mm expansion of this structure, trimmed to chest wall muscle and skin. Seed positions were forward-planned on this scan to deliver the prescribed minimum peripheral dose of 90 Gy using Advantage ${ }^{\mathrm{TM} 103} \mathrm{Pd}$ seeds (IsoAid, Port Richey FL) [7]. Seeds were separated by the defined distances in the treatment plan with absorbable spacers and wrapped in a suture material to cre-

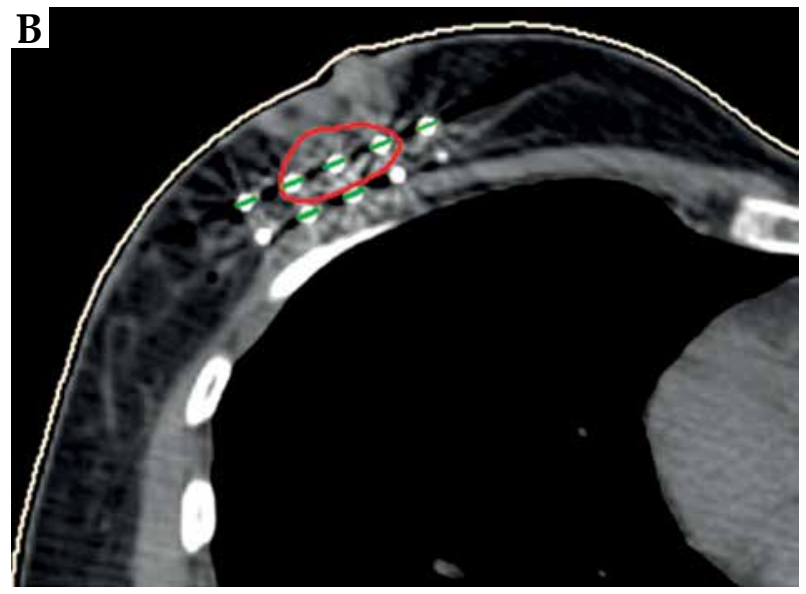

Fig. 1. Simplified schematic of the plan reconstruction process. A) and (B) show an axial slice of the planning and post-implant CT, respectively. The CTV is contoured in red and the body in white. Seeds are indicated in green. C) shows the manual reconstruction (i.e., ground-truth match) for the same patient. Colored dots indicate the planning seed locations, with each needle shown in the same color and connected by dashed lines. Grey squares indicate post-implant seeds. Solid lines connect matched planned and post-implant seed locations. Planned and post-implant seed clouds have been registered using their centers-of-mass 
ate strands in pre-loaded needles. An axial slice showing a typical plan is shown in Figure 1A.

All patients underwent a post-implant CT scan within hours of the implant (i.e., Day 0) in the same orientation as their planning scan [18]. A post-plan was completed on all patients using a similar process to that described by Hilts et al. [17]. Briefly, the post-implant CT scan was deformably registered to the planning $\mathrm{CT}$ scan to obtain the CTV contour, which was then reviewed and adjusted as necessary by a radiation oncologist. Seeds were located on the post-implant CT scan using the automated seed-finding algorithm of the MIM Symphony ${ }^{\mathrm{TM}}$ treatment planning system (MIM Software, Cleveland $\mathrm{OH}$ ). All identified seeds were reviewed and those that were improperly identified were manually adjusted. All seeds were located for all patients. A post-plan axial slice is shown in Figure 1B.

\section{Manual reconstruction}

A manual reconstruction was performed on all patients in the study to define the ground-truth match between each planned seed position and the physically delivered counterpart identified on the Day 0 CT scan. Two observers independently performed the match for each patient. If they did not obtain consistent answers, a third observer independently performed the match. If the third observer obtained a result consistent with one of the previous observers, a meeting was held to reach consensus. If the third observer obtained another unique result, that patient was analyzed separately. An example of a visual representation of a manual match is shown in Figure 1C.

\section{Optimization problem definition}

\section{Simulated annealing overview}

Simulated annealing has been used extensively in radiotherapy, and specifically brachytherapy, optimization $[12,28,29,30]$. The computational process of simulated annealing [31] mimics the physical process of annealing, where molecules in a slowly cooling substance redistribute themselves to reach the lowest energy state. In the computational realization, energy states are described using an objective function and a decreasing temperature parameter dictates the probability of acceptance of energy states. In comparison to gradient descent algorithms, optimization by simulated annealing permits the escape of local minima by probabilistically allowing the acceptance of a worse solution towards the ultimate goal of reaching the global minimum.

As a stochastic optimization algorithm, simulated annealing will not necessarily consistently converge to the global minimum. Thus, the algorithm was run 10 times for each patient to assess this variability. The algorithm was written in MATLAB R2016a (The MathWorks, Inc., Natick, MA, USA)

\section{Initialization}

Plan and post-implant seed clouds were exported from the treatment planning system for each patient. To obtain a consistent co-ordinate system, the centers-of- mass of these seed clouds were initially rigidly registered for the matching process.

\section{Objective function}

In the context of simulated annealing, the objective function defines the quality of a solution. When framed as a minimization problem, a higher value for the objective function indicates a less optimal solution than a lower value. The algorithm is thus designed to move towards lower objective function values. In this application, the objective function describes the optimality of the given implant reconstruction.

The objective function was defined to include two terms: one focused on the distance between matched plan and post-implant seeds, and the other focused on interseed spacing to take advantage of the stranded nature of the seeds used at our clinic. Mathematically, this can be written as:

$$
E=w_{1} \sum_{i=1}^{N}\left|\mathbf{r}_{i}-\mathbf{r}_{i}^{\prime}\right|+w_{2} \sum_{j=1}^{M}\left|s_{j}-s_{j}^{\prime}\right|
$$

where:

$-E$ is the value of the objective function for the given solution,

$-w_{1}, w_{2}$ are empirically-determined weighting factors,

$-N$ is the number of seeds,

- $M$ is the number of interseed spacings (i.e., spacers),

- $\mathbf{r}_{i}, \mathbf{r}_{i}^{\prime}$ are planned and post-implant seed locations, respectively,

$-s_{j}, s_{j}^{\prime}$ are planned and post-implant interseed spacing distances, respectively.

The terms in the objective function were selected based on qualitative clinical assessment of the important parameters that define a 'good' reconstruction. Weighting factors, $w_{1}$ and $w_{2}$, were determined via trial-and-error to be 1 and 4, respectively, and the same factors were used for all patients.

\section{Locality and solution generation}

At each iteration of the algorithm, a new solution was generated by selecting a subset of matches to change. In this application, matches to reassign were probabilistically selected based on their contribution to the overall objective function. As the algorithm neared convergence, the number of matches to reassign was reduced for finer adjustments. When initialized, the algorithm selected five matches to change. This gradually reduced to two as convergence was approached.

Once a solution was generated, each needle was 'unwrapped' or the post-implant seeds were re-matched within the needle in the direction of insertion. This effectively constrained the solution space to physically-feasible matches.

\section{Acceptance probability}

At each iteration, the objective function value for the given solution was compared to the objective function value for the previous iteration. If the new objective function value was lower, it was accepted as the new solution. 
If the objective function value was higher, it could still be accepted with some probability:

$$
P=e^{-\Delta E / T}
$$

where:

$-P$ is the probability of accepting the solution,

$-\Delta E$ is the difference in objective function value between

the new and previous solutions,

$-T$ is the temperature at the given iteration.

This is characteristic of simulated annealing, differentiating it from standard gradient descent algorithms by allowing the escape of local minima.

The temperature parameter decreases as iterations progress. In conjunction with the acceptance probability, this means that early in the optimization, larger 'jumps' in the objective function will be permitted. As the algorithm approaches convergence, the probability of accepting a worse solution decrease.

\section{Geometric implant metrics}

To compute implant metrics, a separate rigid registration was performed on the seed clouds by matching the geometric centroids of the seroma contours on the plan and post-implant CT scans to allow for the calculation of physically relevant implant metrics.

Seed placement accuracy [20,32] or the displacement of the post-implant seed location relative to the planned location were calculated in the needle co-ordinate system. This co-ordinate system is oriented relative to the template and

Table 1. Patient characteristics at planning for patients included in algorithm assessment

Patient characteristics

\begin{tabular}{lc}
\hline Number of patients & 19 \\
\hline CTV volume $\left[\mathrm{cm}^{3}\right]$ & $9 \pm 6$ \\
\hline PTV volume $\left[\mathrm{cm}^{3}\right]$ & $51 \pm 21$ \\
\hline Number of seeds & $65 \pm 17$ \\
\hline Number of needles & $15 \pm 3$ \\
\hline Density of seeds relative to PTV $\left[\right.$ seeds $\left./ \mathrm{cm}^{3}\right]$ & $1.3 \pm 0.2$
\end{tabular}

Values are mean \pm standard deviation

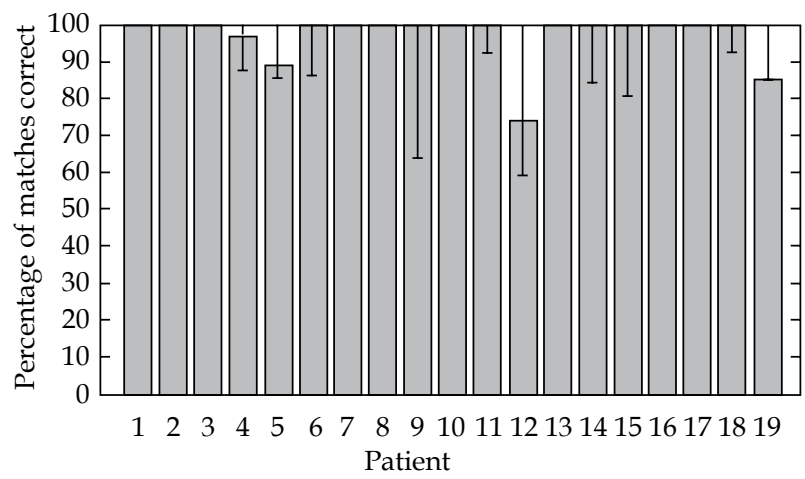

Fig. 2. Algorithm performance for 19-patient cohort. The algorithm was used to reconstruct the implant 10 times for each patient; the bar denotes the mean and the error bar the standard deviation of those runs defines three orthogonal directions: shallow-deep (along the axis of insertion), down-up, and left-right. Trajectory of the needle relative to the planned insertion angle was assessed by averaging the angular deviation between successive seeds along each needle in both the pitch (rotation about the left-right axis) and yaw (rotation about the negative downup axis) directions. Finally, a stretching term similar to that described by Taschereau et al. [24] for permanent prostate seed implant was quantified as the ratio of the post-implant seed train length to the planned seed train length. These metrics have been quantified in the literature for permanent prostate seed implant, and together can provide a comprehensive picture of the relevant geometric quality of an implant. All metrics were characterized for the seed or needle population as a whole and grouped according to seed count ( 2 seed needles to $7+$ seed needles). Statistical analysis to compare metrics between distributions was performed in MATLAB using the Kruskal-Wallis test $(\alpha=0.05)$.

Seed placement accuracy was compared with post-implant dosimetry to assess any correlations. Dosimetric results were calculated using the TG-43 protocol [33] for the dose evaluation volume; a $0.5-\mathrm{cm}$ expansion of the post-implant seroma, trimmed to the chest wall muscle and skin (5-mm inner rind from the body).

\section{Results}

\section{Patient selection}

Twenty-three patients treated at our institution were considered for this study. Independent matches were consistent among two observers for 19 patients. Two of the 19 patients required a third observer to reach a consistent match of two observers and had the third inconsistent observer agree with the match in a second blinded review of the matches in disagreement during a consensus meeting. The reconstruction that was identified as the consensus reconstruction for each patient was defined as the ground truth. For four patients, three independent observers performed the match, and all obtained different results (see Section Inconsistent manual matches). Patient characteristics for the 19 patients with ground-truth matches are shown in Table 1.

\section{Algorithm performance}

The correctness of the algorithm, as measured by the percentage of matches to the correct seed defined in the ground-truth match, for 10 iterations on each of the study patients is demonstrated in Figure 2. Patients are sorted from smallest to largest seed placement error, based on the hypothesis that those with a larger seed placement accuracy (i.e., poorer seed placement) may prove more challenging to reconstruct. Based on the results shown in Figure 2, however, this hypothesis was not proven. The overall accuracy of the algorithm was $97 \pm 8 \%$.

For nine of 19 patients, the algorithm obtained the correct answer (i.e., the ground-truth match) through all 10 iterations. For a further six, the algorithm had a median performance of the correct answer. For three, the algorithm obtained the correct answer at least one time. For one patient (Patient 4), the algorithm obtained a me- 
dian correctness of $96.9 \%$ but never obtained the correct answer. This patient is discussed in further detail in Section Objective function validation. Common mistakes observed in algorithm solutions that did not match the ground-truth include swapping end seeds in needles that curved towards each other and challenges confirming multiple identically-loaded needles implanted in close proximity to each other.

Of particular note in Figure 2 is Patient 12, for whom the algorithm performed the poorest. While this patient had a seed placement accuracy very close to the cohort average $(12 \pm 5 \mathrm{~mm})$, a substantial variation was observed in the accuracy in the left-right direction (ranging from $-12 \mathrm{~mm}$ to $5 \mathrm{~mm}$ ). This patient further had the highest seed count (111) of the 19 patients. These characteristics proved challenging for the algorithm, with observed issues particularly in the left-right direction in the matching.

\section{Objective function validation}

For the 19 patients included in the assessment of algorithm performance, the objective function value for the ground-truth match was equal to or lower than that yielded by the algorithm with the exception of one patient (Patient 4 in Figure 2). For this patient, the reconstruction with the lowest objective function obtained by the algorithm had two post-implant seeds swapped compared to the ground-truth match. Two needles planned $10 \mathrm{~mm}$ apart (in the down-up direction) were both observed pitched upwards. The manual solutions chose the seed arrangements that appeared to have the most realistic pitch, yielding an objective function value $0.14 \%$ higher than that found by the algorithm.
Table 2. Implant metrics for 19-patient cohort (1235 seeds, 283 needles). Right, deep, and up are defined as positive directions; pitch up and yaw right are defined as positive angulations

\begin{tabular}{lc} 
Seed placement accuracy $[\mathrm{mm}]$ & $12 \pm 5$ \\
\hline Left-right & $1 \pm 5$ \\
\hline Shallow-deep & $7 \pm 8$ \\
\hline Down-up & $0 \pm 4$ \\
\hline Trajectory deviation [ $\left.{ }^{\circ}\right]$ & $1 \pm 7$ \\
\hline Pitch & $1 \pm 7$ \\
\hline Yaw & $1.00 \pm 0.03$
\end{tabular}

Values are mean \pm standard deviation

\section{Implant metrics}

A summary of the implant metrics is provided in Table 2. Seed placement accuracy is shown in Figure 3. The greatest misplacement was observed in the insertion direction (i.e., shallow-deep). A statistically significant difference in seed placement accuracy was observed according to seed count in needle $(p<0.001)$ and direction $(p<0.001)$. No statistically significant difference based on seed count in needle was observed for pitch $(p=0.2)$, yaw $(p=0.4)$, or stretching ratio $(p=1.0)$.

The correlation between post-implant dosimetry, measured using the dose evaluation volume $\mathrm{V}_{90 \%}$, and seed placement accuracy is shown in Figure 4. This data is poorly correlated $(R=-0.39)$.
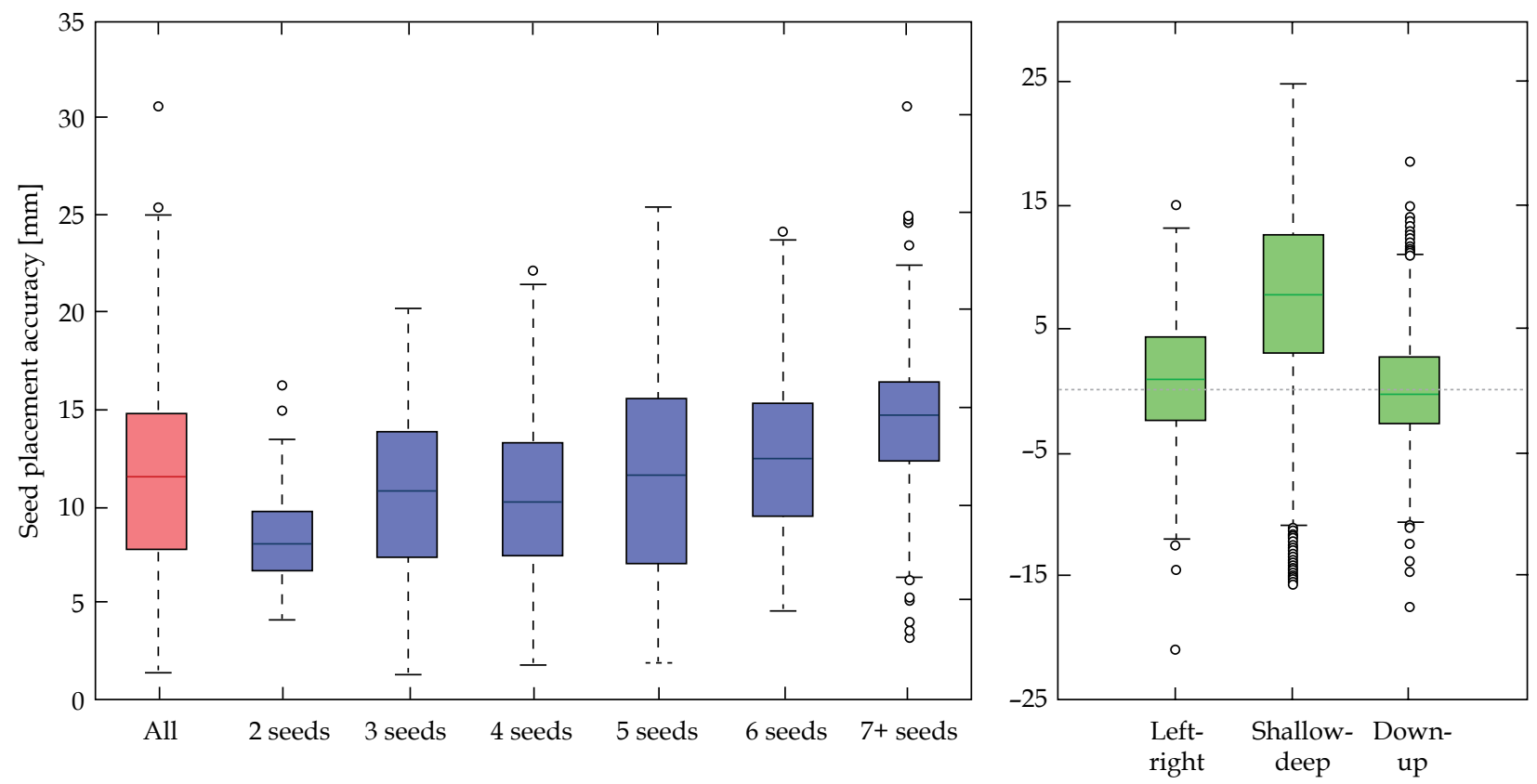

Fig. 3. Seed placement accuracy for the 19-patient cohort (1235 seeds). Accuracy is shown for the entire seed population (red), grouped by number of seeds per needle (blue), and by direction in the needle co-ordinate system (green). Right, deep, and up are defined as positive directions. In this boxplot visualization, the central line denotes the median, the box extends over the interquartile range (IQR; $25^{\text {th }}$ to $75^{\text {th }}$ percentile), the whiskers extend to the most extreme data points excluding outliers, and the outliers (shown as circles) are defined as points $>1.5 \times \mathrm{IQR}$ above or below the $75^{\text {th }}$ or $25^{\text {th }}$ percentiles, respectively 


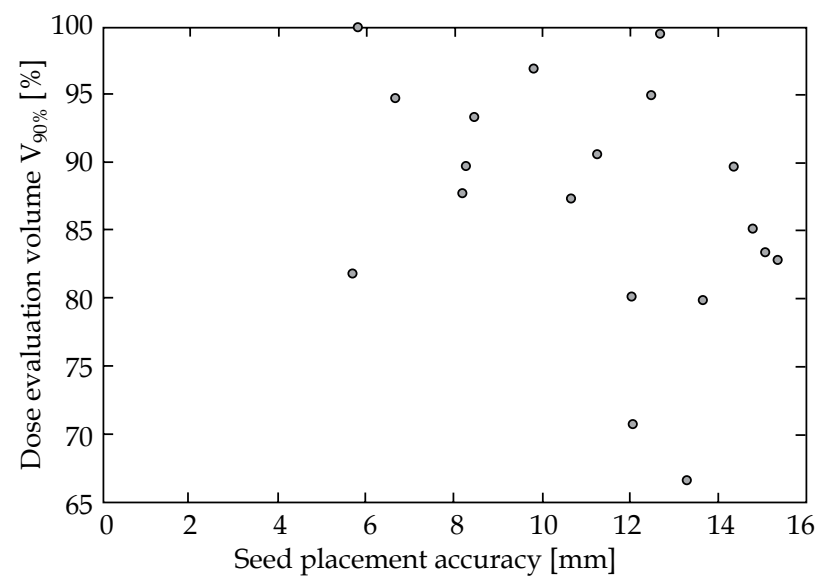

Fig. 4. Assessment of the correlation of the dose evaluation volume (5-mm expansion of the post-implant CTV contour) $\mathrm{V}_{90 \%}$ and the seed placement accuracy for the 19-patient cohort. Poor correlation was observed $(R=-0.39)$

\section{Inconsistent manual matches}

For four patients, three independent observers performed the match and each produced a unique reconstruction; that is, it was not possible to establish a groundtruth match for these patients. Each of these patients had a high seed count $(87,102,109$, and 116 , respectively), and exhibited some needle non-parallelism and overlapping.

For the same four patients, Figure 5 shows the seed placement accuracy determined by the three independent manual reconstructions as well as the algorithm (five iterations of the algorithm were run with the lowest objective function value selected). No statistically significant difference was observed between the seed placement distributions obtained by each observer and the algorithm for each patient; however, placement accuracy on an individual seed basis could vary substantially.

\section{Discussion}

The development of a plan reconstruction algorithm applied to PBSI is a key step towards automating and standardizing post-implant analysis. The ability to easily gather quantitative metrics on implant quality will allow the provision of continual feedback to the brachytherapy team to enhance this treatment technique. Without an automated algorithm, metrics can exclusively be gathered through manual reconstruction, which often necessitates specialized software architecture to view planned and post-implant seed positions and an experienced observer to perform the matches.

In this study, independent observers obtained unique manual reconstructions on four of 23 patients. Such scenarios illustrate the utility of an automated algorithm. Challenging reconstructions tend to be the result of needle overlap or clustering, rendering a manual reconstruction highly subjective and time-consuming to perform. Gathering global statistics on the implant, however, can be highly informative for the brachytherapy team for these patients. In these four patients, the algorithm obtained implant metrics that did not differ significantly from the observers. Thus, this can be used even for such challenging patients, where manual reconstruction may not be feasible. Perhaps even more useful in these situ-

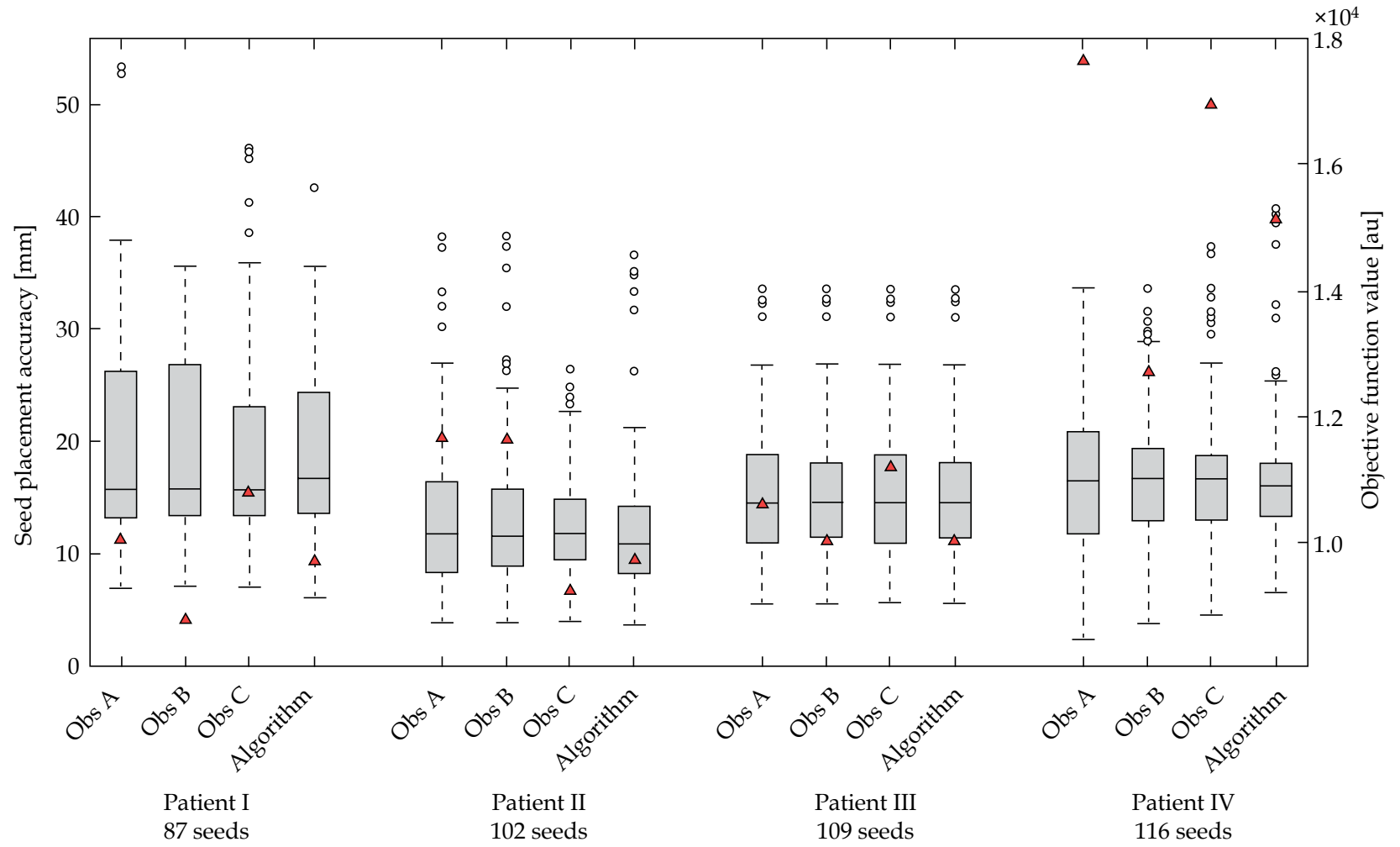

Fig. 5. Seed placement accuracy (left axis; boxplots including outliers) and objective function value (right axis; red triangles) for the four patients for whom each observer obtained a unique manual reconstruction. Patients are ordered by seed count. Boxplot formatting is consistent with Figure 3 
ations, where no ground-truth can be established, is the ability provided by the infrastructure developed for the algorithm to visualize the needle trains to elucidate information on the implant (for example, strands breaking in the chest wall muscle or overlapping with each other, displacing their structure).

Two different plan reconstruction algorithms have been reported in the literature for permanent prostate seed implant. Archambault et al. [22] presented a simulated annealing algorithm that defined the objective function in terms of the minimum distance between registered plan and post-implant seeds. Their results found that the accuracy of the algorithm was highly dependent on seed density. Similar to our results reported here, their study found that even when the algorithm did not yield the correct result (often in the case of high seed density), global statistics on implant quality and accuracy could still be retrieved. Chng et al. [23] presented an S-reconstruction algorithm that used three terms to define similarity to improve upon the robustness of the algorithm: positions of the seeds, angle of the seed trains, and seed train spacing. Accuracy of the algorithm compared to manual reconstruction in their study for 70 cases was $97.7 \pm 0.5 \%$. The accuracy obtained by our algorithm was similar to that reported for both of these permanent prostate seed implant applications.

This current realization of the algorithm enforces that the seed count in the plan matches the seed count in the post-plan. This may be a limitation in some clinical scenarios. Clinics typically order a spare preloaded needle that can be implanted if needed during the procedure in response to changes in patient anatomy from the planning CT scan or other intraoperative decisions to improve dosimetry. Such an implant, however, would represent a deviation in seed count, and thus would require the manual removal of these seeds prior to loading the plan into the algorithm. Further, seed identification may prove challenging for patients with a high number of clips or if the artifact of the seeds overlaps. In this study, all anatomical information was used to identify all seeds to the best extent possible. This can be time-consuming, however, and not necessarily feasible in a clinical setting. Therefore, future improvements to the algorithm may seek to relax this seed count consistency restraint to allow some implanted seeds not to be matched to a corresponding planned seed. This feature has been successfully implemented in plan reconstruction for permanent prostate seed implant, as investigated by Archambault et al. [22]

In 2016, Morton et al. [20] undertook the first investigation of geometric accuracy for PBSI. Their study involved a 20-patient cohort, yielding a seed placement accuracy of $9 \pm 5 \mathrm{~mm}$. Our results are consistent with their clinic's report, while noting deviations in the accuracy in the shallow-deep direction, when comparing the results of their study, $0 \pm 8 \mathrm{~mm}$, to those of ours, $7 \pm 8 \mathrm{~mm}$. There appeared to be a bias towards implanting deep in our cohort. Investigation into potential causes revealed that a systematic offset of seeds due to the bone wax needle plug being accounted for in both planned needle depth and during seed delivery, accounting for a portion of the systematic offset. This discovery underscores the impor- tance of site-specific implant accuracy to evaluate the delivery deviations for each clinic's specific implementation of the technique. Slightly different rigid registration techniques were used for the determination of seed placement accuracy in these studies. Morton et al. [20] performed their registration in the treatment planning system using a 10-mm expansion of the seroma, validating this process by comparison of surgical clip location. In our work, a rigid registration was performed using the centroid of the seroma contours. The analysis of seed placement accuracy was repeated for all patients in our cohort using a rigid registration method similar to that employed by Morton et al. For the population, this resulted in changes of approximately $1 \mathrm{~mm}$ for average directional and total deviations.

Previous work [21] has indicated that the immediate (Day 0) post-implant CT scan may not be the most appropriate time point for evaluating delivered dose. It does, however, have importance for geometric evaluation of the implant. Such metrics can provide invaluable feedback to the brachytherapy team on implant technique, while potentially informing the planning process. In our clinical experience, seed strands tend to break down in the weeks following implant leading to seed coalescence. The magnitude of this coalescence, however, appears to vary among patients. The realization of the algorithm on the Day 0 CT scan highly weighted the interseed spacing term. This may not be appropriate on future time points, where spacing between the seeds is no longer as constrained. Consequently, the objective function may need to be adjusted to apply this reconstruction algorithm to later time points.

\section{Conclusions}

In this work, we present a simulated-annealing-based algorithm for automated plan reconstruction on the immediate post-implant CT scan in PBSI. Tested on 19 of our patients, the algorithm obtained $97 \pm 8 \%$ of the correct matches. This algorithm will facilitate the collection of implant metrics, allowing the timely provision of feedback to brachytherapy teams to improve the clinical process.

\section{Acknowledgements}

The authors would like to acknowledge Dr. Daniel Morton, Dr. Michelle Hilts, and Dr. Deidre Batchelar for helpful discussions. Financial research support for authors was provided by a Canadian Institutes of Health Research (CIHR) Doctoral Research Award (EW) and an Alberta Innovates - Health Solutions (AIHS) Summer Studentship (MS).

\section{Disclosure}

Authors report no conflict of interest.

\section{References}

1. Vicini FA, Kestin L, Chen P et al. Limited-field radiation therapy in the management of early-stage breast cancer. J Natl Cancer Inst 2003; 95: 1205-1210. 
2. Smith BD, Arthur DW, Buchholz TA et al. Accelerated partial breast irradiation consensus statement from the American Society for Radiation Oncology (ASTRO). Int J Radiat Oncol Biol Phys 2009; 74: 987-1001.

3. Polgár C, Van Limbergen E, Pötter R et al. Patient selection for accelerated partial-breast irradiation (APBI) after breast-conserving surgery: Recommendations of the Groupe Européen de Curiethérapie-European Society for Therapeutic Radiology and Oncology (GEC-ESTRO) breast cancer working group. Radiother Oncol 2010; 94: 264-273.

4. Shah C, Vicini F, Wazer DE et al. The American Brachytherapy Society consensus statement for accelerated partial breast irradiation. Brachytherapy 2013; 12: 267-277.

5. Correa C, Harris EE, Leonardi MC et al. Accelerated partial breast irradiation: executive summary for the update of an ASTRO evidence-based consensus statement. Pract Radiat Oncol 2017; 7: 73-79.

6. Shah C, Vicini F, Shaitelman SF et al. The American Brachytherapy Society consensus statement for accelerated partial-breast irradiation. Brachytherapy 2018; 17: 154-170.

7. Pignol JP, Keller B, Rakovitch E et al. First report of a permanent breast $103 \mathrm{Pd}$ seed implant as adjuvant radiation treatment for early-stage breast cancer. Int J Radiat Oncol Biol Phys 2006; 64: 176-181.

8. Pignol JP, Caudrelier JM, Crook J et al. Report on the clinical outcomes of permanent breast seed implant for early-stage breast cancers. Int J Radiat Oncol Biol Phys 2015; 93: 614-621.

9. Keller BM, Ravi A, Sankreacha R, Pignol JP. Permanent breast seed implant dosimetry quality assurance. Int J Radiat Oncol Biol Phys 2012; 83: 84-92.

10. Morton D, Batchelar D, Hilts $\mathrm{M}$ et al. Incorporating three-dimensional ultrasound into permanent breast seed implant brachytherapy treatment planning. Brachytherapy 2017; 16 : 167-173.

11. Frederick A, Meyer T, Roumeliotis M. Treatment planning considerations for permanent breast seed implant. Brachytherapy 2018; 17: 456-464.

12. Roumeliotis M, Yates B, Watt E et al. Demonstration of simulated annealing optimization for permanent breast seed implant treatment planning. Brachytherapy 2018; 17: 615-620.

13. Long K, Karchewski-Welter R, Roumeliotis M et al. 115: Proposal for a Permanent Breast Seed Implant (PBSI) Training Program. Radiother Oncol 2016; 120: S43.

14. Roumeliotis M, Quirk S, Skarsgard M et al. Development and characterization of an anthropomorphic breast phantom for permanent breast seed implant brachytherapy credentialing. Brachytherapy 2018; 17: 506-513.

15. Batchelar D, Hilts M, Rose T et al. Simulation and intraoperative checks for improved standardization and reproducibility of partial breast seed implant technique. Brachytherapy 2014; 13: S84.

16. Karchewski-Welter R, Long K, Watt E et al. 114: Patient positioning and mark-up optimization for permanent breast seed implant (PBSI). Radiother Oncol 2016; 120: S43.

17. Hilts M, Batchelar D, Rose J, Crook J. Deformable image registration for defining the postimplant seroma in permanent breast seed implant brachytherapy. Brachytherapy 2015; 14: 409-418.

18. Watt E, Husain S, Sia M et al. Dosimetric variations in permanent breast seed implant due to patient arm position. Brachytherapy 2015; 14: 979-985.

19. Hilts M, Halperin H, Morton D et al. Skin dose in breast brachytherapy: Defining a robust metric. Brachytherapy 2015; 14: 970-978.

20. Morton D, Hilts M, Batchelar D, Crook J. Seed placement in permanent breast seed implant brachytherapy: are concerns over accuracy valid? Int J Radiat Oncol Biol Phys 2016; 95: 1050-1057.

21. Watt E, Conroy L, Peacock M et al. Appropriate timing for postimplant imaging in permanent breast seed implant: Results from a serial CT study. Brachytherapy 2018; 17: 609-614.

22. Archambault L, Beaulieu L, Tubic D. Automatic post-implant needle reconstruction algorithm to characterize and improve implant robustness analyses. Med Phys 2003; 30: 2897-2903.

23. Chng N, Spadinger I, Morris WJ et al. Prostate brachytherapy postimplant dosimetry: Automatic plan reconstruction of stranded implants. Med Phys 2011; 38: 327-342.

24. Taschereau R, Roy J, Pouliot J. Monte Carlo simulations of prostate implants to improve dosimetry and compare planning methods. Med Phys 1999; 26: 1952-1959.

25. Kaplan ID, Meskell PM, Lieberfarb M et al. A comparison of the precision of seeds deposited as loose seeds versus suture embedded seeds: a randomized trial. Brachytherapy 2004; 3: 7-9.

26. Podder TK, Beaulieu L, Caldwell B et al. AAPM and GEC-ESTRO guidelines for image-guided robotic brachytherapy: Report of Task Group 192. Med Phys 2014; 41: 101501.

27. Jamaluddin MF, Ghosh S, Waine MP et al. Intraoperative factors associated with stranded source placement accuracy in low-dose-rate prostate brachytherapy. Brachytherapy 2017; 16: 497-502.

28. Sloboda RS. Optimization of brachytherapy dose distributions by simulated annealing. Med Phys 1992; 19: 955-964.

29. Pouliot J, Tremblay D, Roy J, Filice S. Optimization of permanent 125I prostate implants using fast simulated annealing. Int J Radiat Oncol Biol Phys 1996; 36: 711-720.

30. Lessard E, Pouliot J. Inverse planning anatomy-based dose optimization for HDR-brachytherapy of the prostate using fast simulated annealing algorithm and dedicated objective function. Med Phys 2001; 28: 773-779.

31. Kirkpatrick S, Gelatt CD, Vecchi MP. Optimization by simulated annealing. Science 1983; 220: 671-680.

32. Frederick A, Watt E, Peacock $M$ et al. Sci-Thur PM Brachytherapy 02: Positional accuracy in Pd-103 permanent breast seed implant (PBSI) brachytherapy at the Tom Baker Cancer Centre (TBCC). Med Phys 2016; 43: 4933-4934.

33. Rivard MJ, Coursey BM, DeWerd LA et al. Update of AAPM Task Group No. 43 Report: A revised AAPM protocol for brachytherapy dose calculations. Med Phys 2004; 31: 633-674. 